\title{
Bettina Biedermann und Heribert Dieter, Hrsg.: Länderbericht
}

Australien. Bonn: Bundeszentrale für politische Bildung, 2012. 450 S. ISBN 978-3-8389-0175-6.

Rezensiert von Fabian Sonnenburg, Universität Köln.

Die Bundeszentrale für politische Bildung bewirbt ihre Länderberichte mit dem Slogan "Die Welt zu sich nach Hause holen". In den bisher erschienenen Sammelbänden geben ausgewählte Autoren dem Leser in verständlicher Sprache umfassende Informationen über Gesellschaft, Mentalität, Wirtschaft, Politik und Kultur des jeweiligen Landes. Der im März 2012 in Bonn von Bettina Biedermann (Kulturanthropologin) und Heribert Dieter (Politikwissenschaftler) herausgegebene Länderbericht Australien folgt diesem Muster. Es ist Ziel des Buchs "Lücken im Kenntnisstand zu schließen und den an Austra-

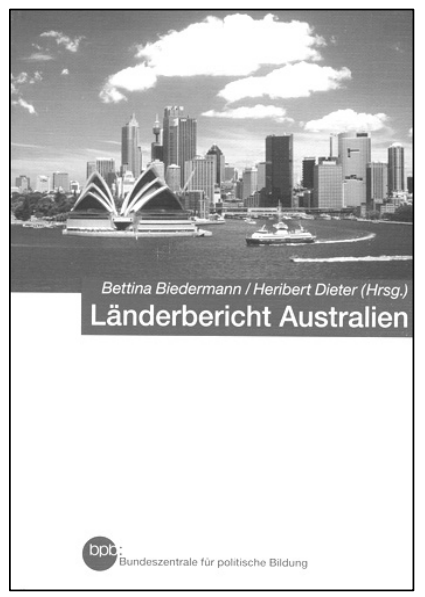
lien interessierten Wissenschaftlerinnen und Wissenschaftlern, aber auch länger nach Australien Reisenden und sich mit Australien beschäftigenden Menschen einen fundierten Einstieg in die Untersuchung der australischen Politik und Gesellschaft [zu] bieten" (18).

Der Band ist in elf Kapitel gegliedert, die sich inhaltlich teilweise überlappen und ergänzen. Da jedes Kapitel ein breites Spektrum an untergeordneten Themen behandelt, können im Rahmen dieser Rezension nur ausgewählte Aspekte Berücksichtigung finden. Den Kern des ersten Kapitels bilden Tabellen, die statistische Kennzahlen der Entwicklung Australiens im internationalen Vergleich zeigen und von Heribert Dieter in groben Zügen interpretiert werden.

Die beiden Herausgeber beschäftigen sich im zweiten Kapitel mit der australischen Geschichte. Nach einem kurzen Abschnitt zur indigenen Bevölkerung geben die Autoren dem Leser Einblicke in die Frühphase der europäischen Besiedlung, die Entwicklung der Strafkolonien und die Erschließung des Landesinneren. Zudem werden historische Meilensteine, wie der Goldrausch, die Gründung des australischen Bundes und Australiens Rolle in den beiden Weltkriegen be- 
handelt. Die Darstellung der Entwicklungen nach 1945 ist auf Einwanderungs-, Wirtschafts- und Außenpolitik beschränkt.

Kate Darian-Smith, eine australische Historikerin, beschreibt im dritten Kapitel die Geschichte und Kultur der beiden indigenen Völker Australiens, der Aborigines und Torres-Strait-Islander, von der britischen Besiedelung bis zur Gegenwart. Diese beiden über Jahrhunderte weitgehend marginalisierten Bevölkerungsgruppen emanzipierten sich in der zweiten Hälfte des 20. Jahrhunderts zunehmend und konnten im Kampf um mehr Selbstbestimmung und Landrechte politische Erfolge verzeichnen. Neuere Gesten der Versöhnung zwischen indigener und nicht-indigener Bevölkerung, wie das Entzünden des Olympischen Feuers durch Cathy Freeman bei den Olympischen Spielen in Sydney 2000 und die offizielle Entschuldigung der australischen Regierung durch den ehemaligen Premierminister Kevin Rudd 2008, berücksichtigt Darian-Smith ebenfalls in ihrem Beitrag.

Im vierten Kapitel erläutert Heribert Dieter die Besonderheiten des politischen Systems. Dabei betont er den Einfluss der britischen und US-amerikanischen Verfassungstradition auf die australische Verfassung. Zudem konkretisiert der Autor die Folgen der relativ schwachen Stellung des australischen Premierministers. Das Rechts- und Regierungssystem, das Wahlsystem und die politischen Parteien bezieht Dieter ebenfalls in seine Ausführungen ein.

Gesellschaft und Kultur Australiens sind Gegenstand des fünften Kapitels von Bettina Biedermann. Sie erörtert die im Ausland wenig wahrgenommenen Selbstdarstellungen und Mythen, die Geschichte der Frauenbewegung, die Vereinbarkeit von Beruf und Familie, die Freizeitgestaltung, das kulturelle Leben sowie Urbanität und Alltag.

Das sechste Kapitel von Heribert Dieter präsentiert die wichtigsten Leitlinien der australischen Bildungs- und Sozialpolitik. Der Autor analysiert die Struktur der staatlichen und privaten Finanzierung des Bildungssystems von der Vorschule bis zur Universität und ordnet seine Leistungsfähigkeit in den internationalen und historischen Kontext ein. Im Bereich der tertiären Ausbildung hebt er die Bedeutung der großen Anzahl an ausländischen Studierenden für die Finanzierung der Universitäten hervor. Der zweite Teil des Kapitels behandelt die Entwicklung des australischen Wohlfahrtsstaats, der sich durch eine minimale Grundsicherung auszeichnet und hohe Anforderungen an die private Absicherung stellt. 
Die Geschichte und aktuelle Entwicklung der Migration und Integration von Einwanderern nach Australien analysiert Bettina Biedermann im siebten Kapitel. Sie erläutert zunächst die Rolle von Häftlingen und freien Siedlern. Ferner geht sie auf die Bedeutung des Goldrausches in den 1850er Jahren ein. Schwerpunkt des Beitrags ist jedoch die traditionell durch starke staatliche Organisation, Finanzierung und Steuerung bestimmte Einwanderungspolitik, welche die gesellschaftliche Entwicklung des Kontinents nachhaltig prägte.

Im Rahmen des achten Kapitels charakterisiert Heribert Dieter aktuelle Strukturen und Entwicklungen der australischen Wirtschaft. Neben Chancen und Risiken des anhaltenden Rohstoffbooms, der Schwäche der verarbeitenden Industrie, dem stabilen Finanz- und dem wachsenden Tourismussektor diskutiert der Autor auch die Reaktionen der australischen Finanzpolitik auf die Weltwirtschaftskrise.

Die Umwelt und Ökologie Australiens ist das Thema des neunten Kapitels. Frank Stilwell, ein australischer Ökonom, geht auf das Spannungsverhältnis zwischen Verstädterung und rohstoffbasiertem Wirtschaftswachstum auf der einen und der Verschärfung umweltpolitischer Maßnahmen auf der anderen Seite ein. Zudem stellt er zentrale umweltbezogene Diskurse und Konflikte vor, weist auf konkrete Fortschritte hin und diskutiert mögliche Entwicklungsmuster.

Das zehnte Kapitel wurde von Benjamin Schreer verfasst und beinhaltet die Entwicklung der Sicherheits- und Verteidigungspolitik. Schwerpunkte sind der Beitrag Australiens in der Bekämpfung des internationalen Terrorismus und die neue Rolle der Volksrepublik China im globalen Machtgefüge. Der Autor erläutert die Prägung der heutigen Politik Australiens durch die historischen Erfahrungen der geographischen Abgeschiedenheit und der damit verbundenen Frage der militärischen Sicherheit. In diesem Zusammenhang betont er die engen sicherheitspolitischen Beziehungen zu Großbritannien und den USA.

Im elften Kapitel evaluiert Heribert Dieter die Stellung Australiens in der Weltwirtschaft. Dabei arbeitet er vor allem die Funktion des Landes als Rohstofflieferant heraus. Weitere Themen sind die zunehmende außenwirtschaftliche Zusammenarbeit Australiens mit asiatischen Staaten sowie die Bedeutung von Wirtschaftsbündnissen. In Bezug auf Konfliktpotentiale und Kooperationen mit asiatischen 
Schwellenländern stellt Dieter die wirtschaftlichen Beziehungen zu China in den Mittelpunkt.

Das zwölfte Kapitel bildet den Anhang. Dieser umfasst eine Zeitleiste, eine Reihe interessanter topographischer und thematischer Karten sowie Hinweise zur weiteren Recherche. Die teilweise kommentierten Literaturverweise am Ende jedes Kapitels können für interessierte Leser von Nutzen sein.

Durch die Konzeption des Sammelbands als Querschnitt durch Struktur und Wesen des Fünften Kontinents ist es den Herausgebern gelungen, eine Vielzahl unterschiedlicher Aspekte abzudecken und dem Leser wenig bekannte Facetten des Landes näherzubringen. Dazu tragen auch die gelegentlichen thematischen Exkurse und Porträts von bedeutenden australischen Persönlichkeiten bei. Viele Sachverhalte werden kritisch hinterfragt und eingeordnet. Dies ist besonders nützlich für Fachwissenschaftler, die an Anregungen aus den Forschungsergebnissen anderer Disziplinen interessiert sind. Inwieweit Leser mit geringem Vorwissen in der Lage sind durch Lektüre dieses Bandes ein übergeordnetes Verständnis für gesellschaftliche Entwicklungen in Australien aufzubauen, ist allerdings fraglich. Dennoch ist die Konzeption des Bandes zu würdigen, da in Anbetracht der inhaltlichen Breite der Ausführungen eine umfassende Verflechtung der Fakten und Erläuterungen zwar erwünscht, jedoch kaum zu leisten ist.

Die Herausgeber setzen einen klaren Schwerpunkt in der Politik und Geschichte Australiens. Dies führt notwendigerweise zur Vernachlässigung anderer Bereiche. Zum Beispiel wird der Entwicklung und den Besonderheiten der australischen Metropolen kein separates Kapitel gewidmet, obwohl Australien eines der am stärksten verstädterten Länder der Erde darstellt. Fast zwei Drittel der Bevölkerung lebte im Jahr 2011 in den fünf großen Verdichtungsräumen Sydney, Melbourne, Brisbane, Perth und Adelaide (ABS 2013). Informationen zu intensiv geführten Diskussionen um die sozialen und ökologischen Folgen der Suburbanisierung tauchen lediglich bruchstückhaft in unterschiedlichen Kapiteln auf. Neben Aspekten der Stadtentwicklung findet auch die landwirtschaftliche Produktion kaum Berücksichtigung. Diese weist zwar nur geringe Anteile an Bruttoinlandsprodukt und Beschäftigung auf, hat aber im Hinblick auf Ernährungssicherheit, Umweltschutz und die Lebensweisen im ländlichen Raum durchaus große Bedeutung für die australische Gesellschaft. 
An vielen Stellen enthält der Text Informationen, die einem Leser, der selbst einen längeren Aufenthalt in Australien plant, von Nutzen sein können (z. B. Gewohnheiten bei der Freizeitgestaltung). Auch stellen die Autoren die Unterschiede zwischen dem bestehenden Bild Australiens in Deutschland und der Realität klar heraus. Komplexe Fachbegriffe werden weitestgehend vermieden oder umgehend im Text erläutert. Der Band spricht folglich sehr unterschiedliche Zielgruppen an. Nicht zuletzt die abwechslungsreiche optische Gestaltung der Seiten mit farbigen Fotos und übersichtlichen Abbildungen trägt dazu bei, dass dieser gelungene Länderbericht zur Faszination der Leserschaft in Bezug auf den Fünften Kontinent beitragen wird.

\section{Bibliographie}

ABS, 2013. 2011 Census of Population and Housing. Canberra: Australian Bureau of Statistics. 